\title{
AN INDUSTRY 4.0 TOOL TO ENHANCE HUMAN-ROBOT COLLABORATION
}

\author{
Athanasios Sidiropoulos, Vasileios Sidiropoulos, \\ Dimitrios Bechtsis \& Dimitrios Vlachos
}
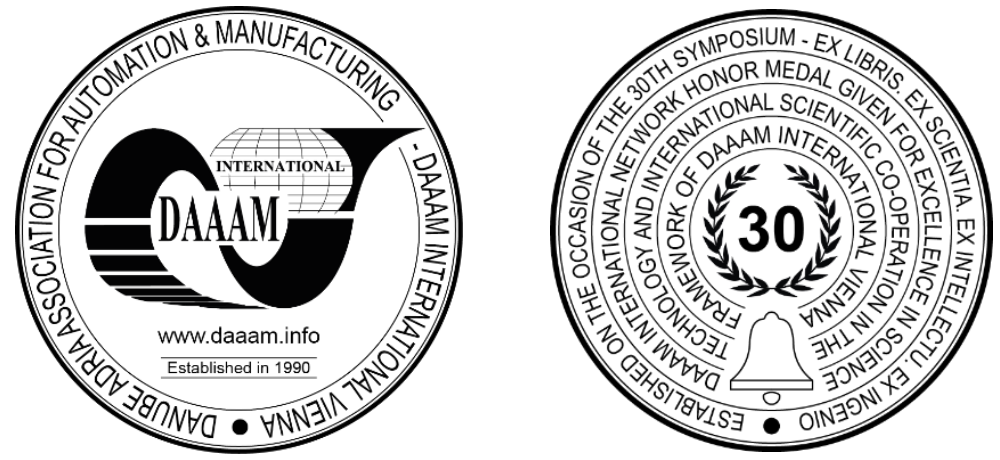

This Publication has to be referred as: Sidiropoulos, A[thanasios]; Sidiropoulos, V[asileios]; Bechtsis, D[imitrios] \& Vlachos, D[imitrios] (2021). An Industry 4.0 Tool to Enhance Human-Robot Collaboration, Proceedings of the 32nd DAAAM International Symposium, pp.0617-0622, B. Katalinic (Ed.), Published by DAAAM International, ISBN 978 3-902734-33-4, ISSN 1726-9679, Vienna, Austria DOI: $10.2507 / 32$ nd.daaam.proceedings.087

\begin{abstract}
The Industry 4.0 is strongly connected with the use of robotic systems and specifically the use of autonomous ground vehicles. A critical issue is the manipulation of the information flows in the industrial facilities and the integration of the robotic systems in the production line. Human Machine Interaction interfaces enable production line employees to monitor in almost real-time the information streams and handle robotic systems and robotic vehicles, but the use of handheld devices is rather limited. In most of the cases the robotic systems are controlled from a central system, and this prohibits the flexibility of the overall system and the re-initialization of the system when an error occurs. The proposed research work focuses on robotic vehicles that are working within the industrial facility and presents a user-friendly Industry 4.0 software tool for transparently monitoring the information streams in the production line and controlling the vehicle's behaviour using mobile devices (e.g., mobile phones and tablets). The developed software tool enables the transparent communication and interaction among the industrial machinery, the robotic systems, and the employees, supports the execution of everyday activities by providing information about the vehicles and assesses the operation of the mobile vehicles.
\end{abstract}

Keywords: ReactJS; web-based ROS tool; human-robot collaboration; Robot Operating System; visualization.

\section{Introduction}

In the era of Industry 4.0 and Industrial Internet of Things (IIoT) the Autonomous Mobile Robots (AMRs) are used in a 24/7 perspective and the human-robot collaboration is a critical issue at industrial facilities. On a recent research it was stated that the drivers of the fourth industrial revolution are automation, big data, cloud computing, Internet of Things (IoT), mass customization and production time improvement [1]. Moreover, as stated by [2] the intelligent autonomous vehicles constitute a radical innovation that could assist in the efficient management of production lines. Information technology tools are extensively used to tackle the above-mentioned issues and transform the industry to a more datadriven approach. Health and safety protocols in industrial facilities and real time location of the robotic vehicles must be managed while the complexity is increasing as new robotic systems are introduced in a daily basis. 
Some researchers developed a safety Industry 4.0 environment as they created a human-centric robotic system navigation in order to ensure the workers' safety within the industrial facilities [3]. The research interest is also focusing on the collaborative tasks that need a human - machine interface for the communication with the industrial robots in the manufacturing sector and thus an online monitoring system is proposed to enhance the collaboration between them, while ensures the security of the executed data transactions [4].

The employees and the autonomous vehicles are moving at the facilities at the 24/7 perspective, and a transparent collaboration framework is necessary for executing common industrial activities. The AMRs are split into 3 categories based on their skills: i) autonomous sensing (information acquisition and data transformation) making observations and refining information, ii) autonomous planning (information interpretation and decision selection) reacting to information or deciding actions and schedule, and iii) autonomous acting (action implementation) executing a planned task or producing reflexive reactions [5]. The autonomous sensing and autonomous planning AMRs enable vehicles to execute tasks in a fully automated manner based on a predefined schedule while the autonomous acting AMRs execute user driven tasks. Robotic vehicles are used at industrial facilities for executing a variety of activities according to their skills. For example, on an assembly procedure the autonomous sensing and autonomous planning AMRs are preferred while in semi products transportation the autonomous acting AMR is the ideal type.

Moreover, in modern industrial facilities and warehouses, picking activities should be executed automatically in order to optimize space and resource utilization and minimize the delivery times. Thus, software tools based on Robot Operating System (ROS) for time and distance efficient AMR routing across multiple points in industrial facilities have been proposed [6]. Furthermore, there are several tools for the visualization of ROS activities while, RViz is the dominant built-in graphical tool [7] for visualizing data-streams from the sensors of the AMR. Indicatively, RViz presents data obtained from cameras, laser range finders, inertial measurement units, and many more [8]. However, RViz needs direct access to the AMR while it cannot work on systems without a preconfigured ROS environment.

The proposed solution is developed on top of the ROS platforms and TurtleBot2 is used for validating the developed tool in real-world scenarios. ROS is an open-source meta-operating system with a collection of sophisticated tools that can be used for enabling the scheduling and planning activities of mobile robots at industrial environments [6]. The proposed web-based software informs the end-user about: (i) the position of the AMR in the facility using the Occupancy Grid Map (OGM), (ii) the task that robot executes, while it enables the capabilities of (iii) on-line AMR programming and (iv) reinitialization of the robots' parameters, as the disorientation of the AMRs is a big issue.

The rest of the paper is structured in 4 sections. Section 2 analyses the research methodology and the background of the human-robot collaboration issues and solutions. Section 3 explains the proposed software tool that implemented for: (i) human-robot collaboration enhancement and (ii) robot on-line programming. Finally, the Section 4 ends up with conclusions and further research.

\section{Literature Review}

The Industry 4.0 is the main trend at manufacturing as the use of Information Systems, IoT, cloud, big data and artificial intelligence is rapidly increasing. A research concluded that IoT is an enabler for Industry 4.0 and smart manufacturing while it has privacy/security issues that need to be addressed in order to ensure the secure access of the data streams to the employees [9]. Moreover as stated by [10], enterprise information systems (EISs) will benefit from Industry 4.0. Specifically, new types of advanced manufacturing and industrial processes revolving around machine-tohuman collaboration and symbiotic product development will emerge. In industries the main goal is to be more competitive by minimizing costs and maximizing their efficiency and effectiveness while for operational cost reduction they tend to use more robotic systems. Thus, information technologies can help industries by adding more robotic systems and AMRs in their production lines.

A common problem that AMRs are facing in a daily basis is the obstacle avoidance, while more robots are constantly added on the existing facilities and humans are also moving within the facilities, the space utilization of the industrial area is increasing. In this overloaded environment, human-centric robot navigation approaches have been published in order to enhance the safety of the employees in the facilities [11]. These approaches are based on Artificial Intelligence (AI) and Machine Learning (ML) and the safety cannot be fully ensured. Thus, a backup tool that allows the user to access the operation of the robot is mandatory. On the safety improvement the ISO 15066 and ISO 18646-2 standards provide guidance on human-robot collaboration and robot navigation inside the industrial facilities by dividing the industrial area into three pieces, namely: robot workspace, robot navigation space and robot collaborative space. The division of the facility area enhances the safety of the employees but adds limitations on human-robot collaboration. Additionally, as stated by [12], the localization of AMRs is a critical issue and the authors proposed cooperative localization using Robotic Swarms. Moreover, an online monitoring tool based on ROS and ReactJS has been developed and the authors concluded that it's an easy to deploy tool as it is supported by all the available web browsers [8]. Other researchers proposed a solution on optimal trajectory problem with affective algorithms where multi-robot (robot swarms) cooperation can be achieved [13].

In modern robotic systems, every established manufacturer has developed proprietary controllers and software tools where off-line programming tasks (OLP) could be executed. The term OLP refers to the technique in which the programming of the robotic tasks is defining before its activation. On the other side the On-line programming technique means the programming of the robotics tasks in real-time. 
In case of AMRs where their task programming is affected by many factors the real-time human interference is obligatory. A research about assembly tasks coordination between humans and robotic systems has been conducted. They simulated a ROS based robot and they managed to enable some features of the on-line programming technique through a developed Graphical User Interface (GUI) as they were able to change the executed tasks in real-time [14]. As a result, when the collaboration between human and robots is the main objective, a tool for on-line programming of the robotic vehicles must be developed in addition to the existent OLP technique as the access to the AMR's operation is an issue that needs to be addressed.

All the above-mentioned limitations exist for quite long time and software tools like RViZ are used to facilitate these shortfalls. While the Web-based applications and services are growing rapidly a new approach for a web-based software tool is proposed and developed for the enhancement of the human-robot collaboration and human's safety within the industrial facilities.

\section{Proposed Solution}

This manuscript proposes a software tool for visualizing the AMR's location in the facility, the dynamic dispatching of the AMRs for executing specific tasks and the AMR's re-initialization, using a user-friendly Graphical User Interface (GUI) implementation that interconnects robotic systems with the employees in the industrial facility. The proposed software tool uses: (i) ROS to control the AMR units, (ii) ReactJS library to plot the visualization map on a webpage, (iii) rosbridge-server as a middleware between Robot units and Web-Based GUI, (iv) nodeJS to create the backbone server and the authentication mechanism and (v) MySQL to save the users' and robots' data.

At Fig. 1 the system architecture of the proposed software tools is presented. The system consists of four main modules: (i) the ROS that controls the robot and all the necessary topics, actions, and messages, (ii) the front-end ReactAPI that creates the webpages for the end-users, (iii) the nodeJS server that enables the access to the database and (iv) the database that contains the users' and robots' data. The main purpose of the React API module is the creation of the GUIs and it is based on the React library. React allows the direct creation of objects that contain and manipulate data without strictly defining how they are used. The basic building block of a React application is the React Component class. React views the UI as a state machine and renders all of its components with a particular state. Finally, the react library connects the created components and generates a multi-page working API. The npm package and yarn package managers are also used to control JavaScript packages while the "GUI data Request" and "authentication Request" routines were implemented to access the database data through the Node JS server. Moreover, on Fig. 1, the ROS JavaScript library (ROSLIBJS) is presented which is responsible for message transactions between web pages and ROS. ROSLIBJS is a library that transforms the ROS messages on a JSON format and transmits them to the ROS environment through the rosbridge module.

From the side of the ROS, the rosbridge server was used which is a middleware abstraction layer that provides robot information to application programmers who are not themselves roboticists [15]. Moreover, the rosbridge protocol as a general relies on ROS topic messaging in a client-server paradigm suitable for wide area networks, and human-robot interaction at a global scale through modern web browsers [16]. The interconnection among ROSLIBJS and rosbridge was used to provide the access of the ROS's actions and topics to the front-end react API.

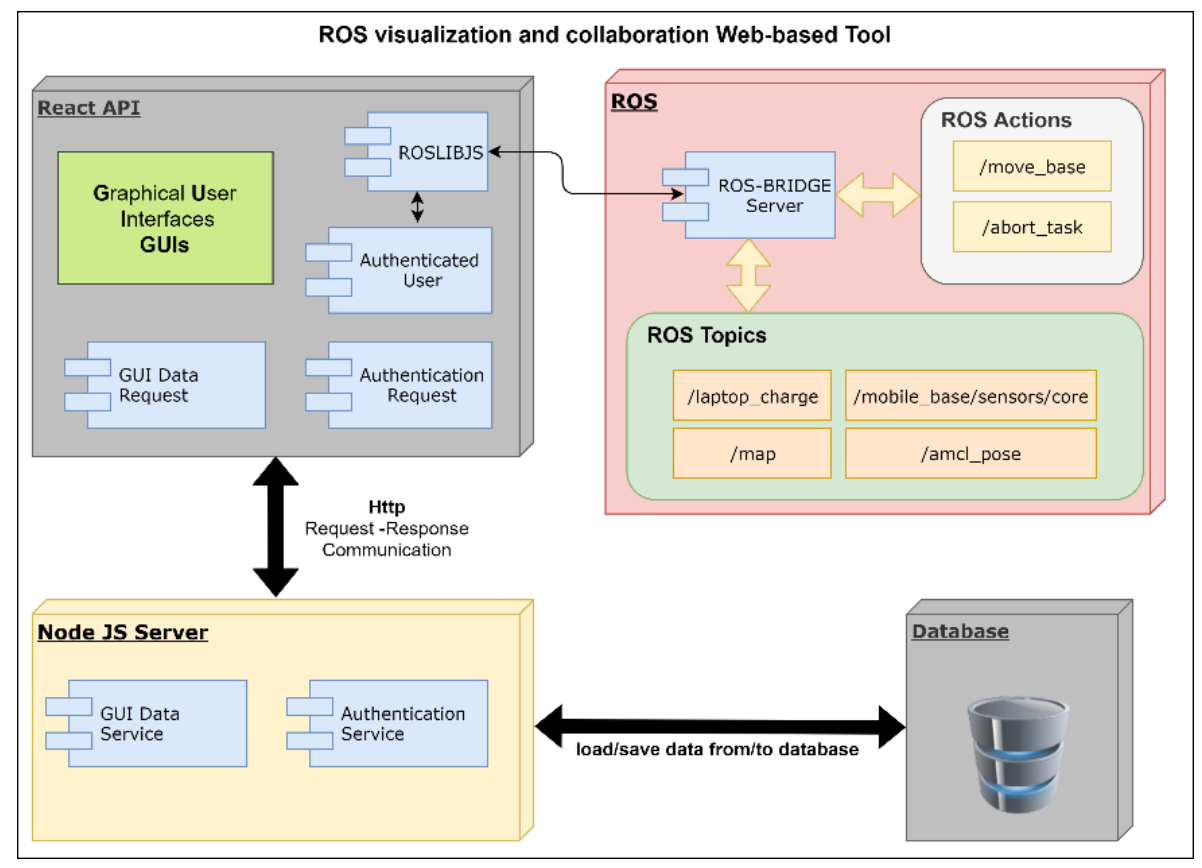

Fig. 1. System Architecture 
Thus, the react library can assign new ROS messages by communicating with the ROS and getting response messages. The following ROS topics used to get the desired information from the robot: (i) "/laptop_charge" topic which provides the battery level of the laptop that we used on our implementation, (ii) "/map" topic that includes the information about the OGM, (iii) "/mobile_base/sensors/core" topic that provides information for the Kobuki® base such as its battery level and (iv) "/amcl pose" topic that has all the information about the current location of the AMR. Also, the ROS actions "/move_base" and "/abort_task" were used for the real-time end-user informing. Specifically, the action "/move base" used for the movement of the robot to a certain location and the "/abort task" action was used to enable the ability of real-time task's cancelation in case of emergency. Moreover, there are the MySQL Database and the Node JS server modules which were used to ensure the privacy and security of the saved data as only an authenticated user can access the system's data. MySQL is an open-source relational database management system that organizes the data into tables while the Node JS server has direct access to the database and exports only the desired data to the external users.

From the perspective of the software web tool the ReactAPI and NodeJS are collaborating to create a full-stack webbased API. The React library is used as a client-side application while the nodeJS used as a server-side script. As mentioned before, the only way that the front-end side can access the database's data is by using http requests from the authenticated users, providing the token that the server sends on the authentication procedure. The developed system shares the information about the AMR only to authenticated users. Thus, the flow, to access the robot information, is analysed as follows: (i) the registered user must enter his username and password on the Log In page (Fig. 2), (ii) the backbone mechanism (server) retrieves user's data from the database and authenticates the user (if he has provide the correct username and password), (iii) the system provides access of the ROSLIBJS to the webpage with the data that retrieve from server and (iv) the system presents the robot information on the webpage while, the user can interact with the AMR and assign new tasks. The available tasks that an authenticated user can assign to AMR are: (i) move the robot to a certain position, (ii) abort the task that is executed in case of emergency, (iii) initialize the robot position and orientation on the map and (iv) assign new tasks. Moreover, the assignment of the tasks is implemented through ROS actionlib package, that enables users to cancel the request or to get periodic feedback about the progress of the assigned task. This package provides tools to create servers that execute long-running goals that can be pre-empted. For the demonstration of the created software tool the prerequisite is a map of the robot's working area. Furthermore, the tool can help the authenticated user to the mapping process as it gives the capability to the end-user to move the robot by clicking on the map area on the webpage. After the map is completed, the API enables the AMR's visualization, and the user monitors the AMR's executed tasks.

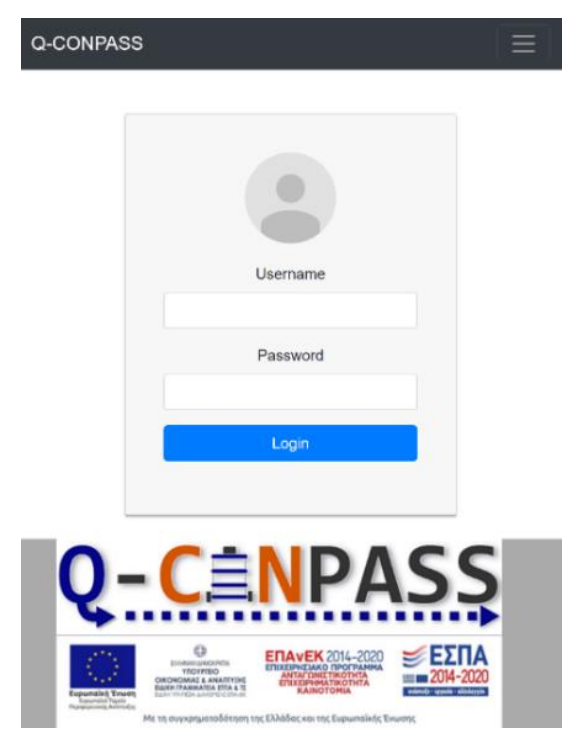

Fig. 2. Login Page of the proposed software tool

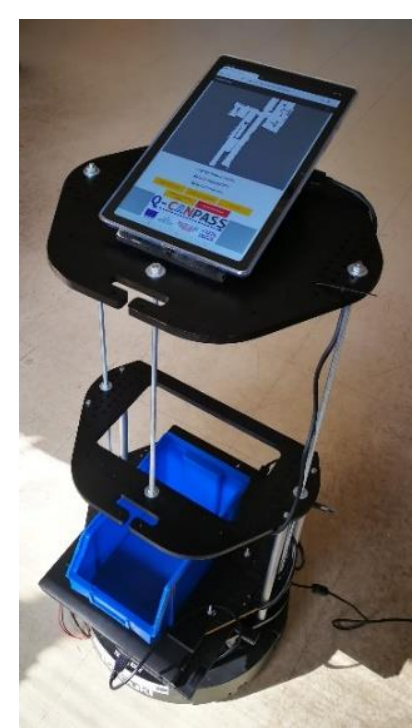

Fig. 3. Developed robot with Tablet and kobuki base

The user's webpage of the proposed software tool is presented at Fig. 4 after the user's authentication procedure. On this page the OGM of the Laboratory of Statistics and Quantitative Analysis Methods and the current position of the robot is presented on the left side of the webpage, while the system provides additional information about the robot on the right side such as robot status, robot's base battery level and laptop's battery level that runs the ROS. Moreover, with the buttons the user can assign the above-mentioned tasks to the robot units. When the user clicks a button, the green colour indicates that the next click on the OGM will assign the defined task to the robot through the action server package. While the robot is moving the application updates the robot's cursor on the map and pop-up messages inform the user for the progress of the executed task through the action server that is running on the ROS. The red button named "Abort execution task" can abort the executed tasks instantly in case of an emergency or a failure that is observed by an employee within the industrial facility. 
Moreover, on Fig. 3 the main parts of the developed robot are presenting, namely: (i) the Kobuki base, (ii) the Slamtec ${ }^{\circledR}$ RPlidar which is located below the laptop's rack, (iii) the laptop with GPU, (iv) the tablet that we used for controlling the robot through the developed software tool and (v) the bin box for the placement of small items. Thus, the robot can move to user-defined points within the industrial facility and transport small parts among employees and workstations. This ability reduces employees' transportation times while enhancing human-robot collaboration as it creates a more flexible and efficient production line.

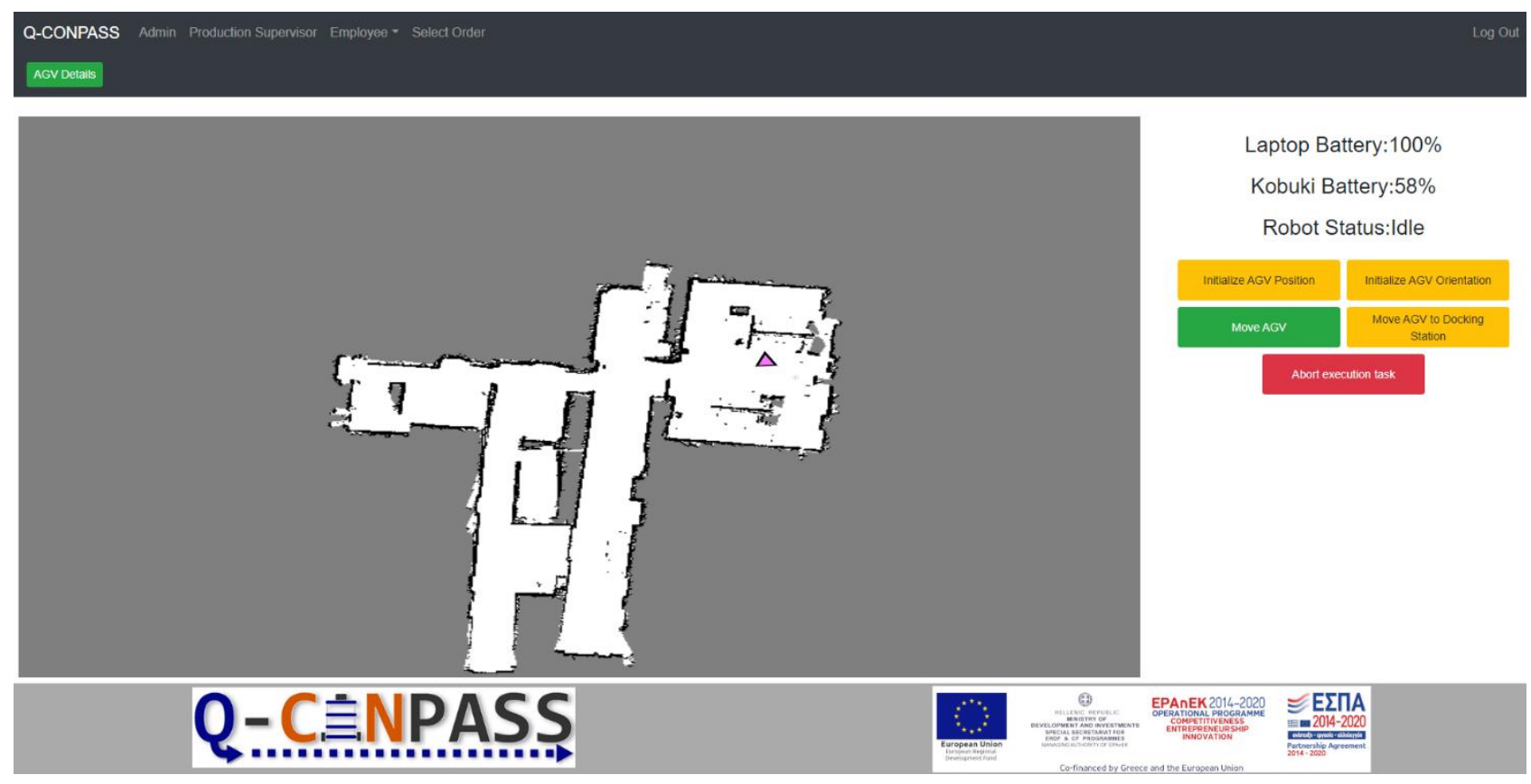

Fig. 4. Webpage with Robot OGM visualization

\section{Limitations and Conclusions}

The development and testing of our proposed solution took place at the laboratory of Statistics and Quantitative Analysis Methods and that set some limitations on our results, as the available area was way smaller than an industrial area and the number of the available end users was also limited. Moreover, the proposed solution tested without taking into consideration robot-user interconnection problems that can occur in a large industrial area.

While robotic systems and AMRs are vastly adopted by industries, the development of tool for user-friendly humanmachine interfaces that allow employees to directly handle the robotic systems in the production line, and to be dynamically informed about the AMR's status, as well as the status of the executed activities is still in an infancy state and should be further examined. The proposed user-friendly software tool addresses some open issues in industrial environments as it can (i) move the AMR during the mapping procedure in the industrial area, (ii) visualize in real-time the industrial area, (iii) assign new tasks to AMRs such as the transportation of small items within the industrial facility and (iv) visualize the position of the AMRs on the OGM. The solution was designed with the use of Kobuki, a small size Turtlebot 2 series mobile robot, but due to openness of ROS it can be used also in all ROS based robots. Also, due to the web-based nature of the proposed tool, it categorized as an easy-to-use software tool in industrial areas, through mobile phone and tablets, when access to a computer is impossible.

The proposed software tool could be further developed to improve its functionality. The next step is the prioritization of the tasks assigned by an end-user according to her authorization role. Thus, a high-level user will have priority on task assignment in comparison with an production line employee. Moreover, a semantic mapping and the separation of the industrial area into divisions would help to add speed limits on the AMRs according to the human activity on each division. Thus, on divisions with low human activity, the AMRs could be moved faster and reduce transportation times while on divisions with high human activity the lower speed limits can enhance safety and assist human-robot collaboration.

\section{Acknowledgments}

This paper was partially supported by the Greek Secretariat of Research and Technology, Operational Programme Competitiveness, Entrepreneurship and Innovation 2014-2020, project "Dynamic Quality CONtrol on Production lines using intelligent AutonomouS vehicleS (Q-CONPASS)". 


\section{References}

[1] Roblek, V.; Meško, M.\& Krapež, A. (2016). A Complex View of Industry 4.0, SAGE Open, Vol. 6, No. 2. doi:10.1177/2158244016653987

[2] Bechtsis, D.; Tsolakis, N.; Vlachos, D.\& Srai, J. S. (2018). Intelligent Autonomous Vehicles in digital supply chains: A framework for integrating innovations towards sustainable value networks, Journal of Cleaner Production, Vol. 181, 60-71. doi:10.1016/j.jclepro.2018.01.173

[3] Papa, M.; Kaselautzke, D.; Radinger, T. \& Stuja, K. (2017). Development of a Safety Industry 4.0 Production Environment, Proceedings of the 28th DAAAM International Symposium, pp.0981-0987, B. Katalinic (Ed.), Published by DAAAM International, ISBN 978-3-902734- 11-2, ISSN 1726-9679, Vienna, Austria, DOI: 10.2507/28th.daaam.proceedings.136

[4] Kuts, V.; Sarkans, M.; Otto, T. \& Tähemaa, T. (2017). Collaborative Work Between Human And Industrial Robot In Manufacturing By Advanced Safety Monitoring System, Proceedings of the 28th DAAAM International Symposium, pp.0996-1001, B. Katalinic (Ed.), Published by DAAAM International, ISBN 978-3-902734-11-2, ISSN 1726-9679, Vienna, Austria, DOI: 10.2507/28th.daaam.proceedings. 138

[5] Stubbs, K.; Wettergreen, D. \& Hinds, P. J. (2007). Autonomy and common ground in human-robot interaction: A field study, IEEE Intelligent Systems, Vol. 22, No. 2, 42-50. doi:10.1109/MIS.2007.21

[6] Karamanos, X.; Mallioris, P.; Poulimenos, D.; Bechtsis, D. \& Vlachos, D. (2019). A ROS Tool for Optimal Routing in Intralogistics, Proceedings of the 30th DAAAM International Symposium, pp.0832-0838, B. Katalinic (Ed.), Published by DAAAM International, ISBN 978-3-902734-22-8, ISSN 1726-9679, Vienna, Austria, DOI: 10.2507/30th.daaam.proceedings. 115

[7] Kam, H. R.; Lee, S. H.; Park, T. \& Kim, C. H. (2015). RViz: a toolkit for real domain data visualization, Telecommunication Systems, Vol. 60, No. 2, 337-345. doi:10.1007/s11235-015-0034-5

[8] Ivanov, A.; Zakiev, A.; Tsoy, T. \& Hsia, K. (2021). Online Monitoring and Visualization with ROS and ReactJS, $0-3$

[9] Thoben, K. D.; Wiesner, S. A. \& Wuest, T. (2017). "Industrie 4.0" and smart manufacturing-a review of research issues and application examples, International Journal of Automation Technology, Vol. 11, No. 1, 4-16. doi:10.20965/ijat.2017.p0004

[10] Lu, Y. (2017). Industry 4.0: A survey on technologies, applications and open research issues, Journal of Industrial Information Integration, Vol. 6, 1-10. doi:10.1016/j.jii.2017.04.005

[11] Tan, Q.; Tong, Y.; Wu, S. \& Li, D. (2019). Anthropocentric Approach for Smart Assembly: Integration and Collaboration, Journal of Robotics, Vol. 2019. doi:10.1155/2019/3146782

[12] Pires, A. G.; Rezeck, P. A. F.; Chaves, R. A.; Macharet, D. G. \& Chaimowicz, L. (2021). Cooperative Localization and Mapping with Robotic Swarms, Journal of Intelligent and Robotic Systems: Theory and Applications, Vol. 102, No. 2. doi:10.1007/s10846-021-01397-z

[13] McLurkin, J. \& Yamins, D. (2005). Dynamic task assignment in robot swarms, Robotics: Science and Systems, Vol. 1, 129-136. doi:10.15607/rss.2005.i.018

[14] Tsarouchi, P.; Makris, S.; Michalos, G.; Matthaiakis, A. S.; Chatzigeorgiou, X.; Athanasatos, A.; Stefos, M.; Aivaliotis, P. \& Chryssolouris, G. (2015). ROS based coordination of human robot cooperative assembly tasks-An industrial case study, Procedia CIRP, Vol. 37, 254-259. doi:10.1016/j.procir.2015.08.045

[15] Crick, C.; Jay, G.; Osentoski, S. \& Pitzer, B. (n.d.). Rosbridge: ROS for Non-ROS Users, $493-504$. doi:10.1007/978-3-319-29363-9

[16] Toris, R.; Kammerl, J.; Lu, D. V.; Lee, J.; Jenkins, O. C.; Osentoski, S.; Wills, M. \& Chernova, S. (2015). Robot Web Tools: Efficient messaging for cloud robotics, IEEE International Conference on Intelligent Robots and Systems, Vols. 2015-Decem, 4530-4537. doi:10.1109/IROS.2015.7354021 